Portland State University

PDXScholar

6-14-2019

\title{
Use of Connectotyping on Task fMRI Data Reveals Dynamic Network Level Cross Talking During Task Performance
}

\author{
Valeria Vazquez-Trejo \\ Portland State University \\ Binyam Nardos PhD \\ Oregon Health \& Science University \\ Bradley Schlaggar MD, PhD \\ Kennedy Krieger Institute \\ Damien Fair PA-C, PhD \\ Oregon Health \& Science University \\ Oscar Miranda-Dominguez PhD \\ Oregon Health \& Science University
}

Follow this and additional works at: https://pdxscholar.library.pdx.edu/honorstheses

Let us know how access to this document benefits you.

\section{Recommended Citation}

Vazquez-Trejo, Valeria; Nardos, Binyam PhD; Schlaggar, Bradley MD, PhD; Fair, Damien PA-C, PhD; and Miranda-Dominguez, Oscar PhD, "Use of Connectotyping on Task fMRI Data Reveals Dynamic Network Level Cross Talking During Task Performance" (2019). University Honors Theses. Paper 740.

https://doi.org/10.15760/honors.757

This Thesis is brought to you for free and open access. It has been accepted for inclusion in University Honors Theses by an authorized administrator of PDXScholar. Please contact us if we can make this document more accessible: pdxscholar@pdx.edu. 
Use of connectotyping on task fMRI data reveals dynamic network level cross talking during task performance.

\author{
by \\ Valeria Vazquez-Trejo
}

An undergraduate honors thesis submitted in partial fulfillment of the

requirements for the degree of
Bachelor of Science
in
University Honors
and
Biology

Thesis Advisors

Oscar Miranda-Dominguez PhD \& Binyam Nardos PhD

Portland State University 


\section{Introduction:}

Task-based functional MRI (fMRI) has had a profound impact on our understanding of brain functioning. Using this non-invasive neuroimaging method, it is possible to design experiments that target specific sensorimotor, perceptual, or cognitive operations in efforts to understand the brain basis of those functions. Complementing neuroscientific findings based on other methods (e.g. single cell or multiunit recording), and lesion cases, task-based fMRI studies have identified the functional neuroanatomy underlying various sensorimotor/perceptual systems such as visual (Engel et al., 1994; Goebel, Khorram-Sefat, Muckli, Hacker, \& Singer, 2001; Sereno et al., 1995) and auditory systems (Moerel, De Martino, \& Formisano, 2014), as well as systems associated with higher order cognitive operations such as memory retrieval (Cabeza, Ciaramelli, Olson, \& Moscovitch, 2008; Dobbins \& Wagner, 2005; Nelson et al., 2010; Rugg \& Vilberg, 2013; Wheeler \& Buckner, 2003; Yonelinas \& Levy, 2002; Yonelinas, Otten, Shaw, \& Rugg, 2005), semantic processing (Badre, Poldrack, Paré-Blagoev, Insler, \& Wagner, 2005; Donaldson, Petersen, Ollinger, \& Buckner, 2001; Fiez, 1997; Friederici, Opitz, \& von Cramon, 2000; Gordon et al., 2016; Petersen, Fox, Posner, Mintun, \& Raichle, 1988; Roskies, Fiez, Balota, Raichle, \& Petersen, 2001; Thompson-Schill, D’ Esposito, Aguirre, \& Farah, 1997; Wagner, Paré-Blagoev, Clark, \& Poldrack, 2001) and cognitive control (Botvinick Todd S Braver et al., 2001; Braver \& Barch, 2006; Dosenbach et al., 2006; F Dosenbach et al., 2007).

The primary measure in $\mathrm{AMRI}$ studies is the blood oxygen level dependent (BOLD) signal. Although not a direct measure of neural activity, it has been shown that the measured BOLD signal is correlated with neural activity, particularly with local field potentials (Logothetis, 2003; Logothetis, Pauls, Augath, Trinath, \& Oeltermann, 2001). The BOLD signal, however, is slow compared to neural activity. After an initial stimulus it takes approximately 20 seconds for the BOLD signal to return to baseline, this observed activation trend is the hemodynamic response function. The peak activation of the BOLD signal typically observed 6 seconds after stimuli presentation (Vazquez \& Noll, 1998). The signal delay in returning to baseline needs to be considered in experimental design. For example, in a typical task experiment, participants are exposed to a given stimulus (visual or auditory) or are asked to perform a task. Given the knowledge of the delayed peak on activation, methods are tuned to look for brain areas that respond specifically to the experimental paradigm once peak response is achieved.

The subsequent development of resting state functional connectivity MRI (rs-fcMRI) was another milestone in neuroimaging. Biswal et al.'s seminal work (Biswal, Zerrin Yetkin, Haughton, \& Hyde, 1995) established that the low frequency $(<0.1 \mathrm{~Hz})$ resting BOLD activity in brain regions that are typically coactivated during task-states (or known to be members of a common brain system e.g. left and right primary motor cortex) shows a high degree of temporal correlation. This high degree of correlation is hypothesized to be a correlate measure of the functional connectivity among the said regions. rs-fcMRI has since become a very convenient technique to characterize brain function because, since it doesn't require the presence of an overt cognitive task, it could be employed in animals, developmental populations, or in patients that may otherwise be unable to perform intentional cognitive tasks.

One limitation commonly shared by both task-based and resting state fMRI is that, due to the noisy nature of the fMRI signal, many trials or functional runs are acquired and subsequently averaged, both within and across subjects, to get a reasonable estimate of the functional measures 
under consideration. This limitation has several consequences, the obvious one being the cost, in terms of both time and money, of collecting a sizeable dataset. The other consequence of this limitation is that averaging is required across many trials and subjects effectively erasing individual differences and potentially informative moment-to-moment or dynamic functional information. For instance, one may reasonably hypothesize that there are dynamic functional connectivity changes on the systems supporting a mental process that may occur on the order of seconds during the instantiation, computation, and response frame of a given task. The noted limitations have to date precluded worthwhile approaches in testing the aforementioned hypothesis.

Connectotyping, a computational approach recently developed in our lab (Miranda-Dominguez et al., 2014), has been demonstrated to have the potential to address the above limitations and allow characterization of dynamic cross-region and cross-network connectivity when applied to rs-fcMRI datasets. Here, we aim to demonstrate that the same technique can be applied to a task fMRI dataset to track changes in network-network functional connectivity during the progression of a task.

The connectotyping approach rests on a simple linear model that proposes that the activity of a given brain region can be described by the weighted sum of other brain regions. The resulting beta coefficients correspond to a personalized model-based "connectotype" beta matrix that is capable of predicting the timeseries of each subject. Connectotyping is an effective way to depict unique patterns of brain connectivity in individuals because it optimizes the signal to noise ratio intrinsic to fMRI allowing us to characterize heritable patterns of brain connectivity (Miranda-Dominguez, Feczko, Grayson, Walum, Nigg, Fair, et al., 2018). This optimization allows connectivity to be defined using a relatively small amount of data (e.g. 5 minutes of rsfcMRI), which is the typical amount of movement-free data able to be acquired in most studies. In addition to allowing for a better characterization of individual differences, the approach also allows us to capture dynamic temporal changes occurring at the time scale of seconds during task progression.

As noted above, the aim of the current study is to determine, as demonstrated using resting state datasets, if connectotyping can be used to track individualized changes in brain connectivity using a task fMRI dataset. To do this, we first identified a task fMRI dataset without the confounding effect of BOLD activity from several past stimuli shown at each time point. As noted above, one of the characteristics of the BOLD signal that must be considered in this context is that it takes about 20 seconds for the hemodynamic response function to return to baseline following stimulus presentation. If stimuli are not presented in a widely-spaced manner, then mathematical modeling would need to be applied to separate the BOLD activity corresponding to each stimulus. However, we avoid this by using data from widely spaced eventrelated fMRI experiment (at least 20 seconds between individual stimuli) in which subjects were performing a visually presented word vs. pronounceable nonword (hereafter pseudoword or PW) lexical decision task (Nardos, 2015).

After having calculated individualized connectyotypes at each time point (i.e. frame or functional run corresponding to $1 \mathrm{TR}$ ) of the experiment, we used a 2 -way repeated measures ANOVA using time point ( 8 frames corresponding to about 20 seconds), stimulus type (word vs. PW), and 
their interaction to identify potentially distinct dynamic functional relations during word vs. PW processing. We hypothesized that dynamic connections between networks implicated in cognitive control (Botvinick et al., 2001; Dosenbach et al., 2006; 2007; Braver \& Barch, 2006), memory retrieval (Iidaka, Matsumoto, \& Nogawa, 2006), and semantic processing(Badre et al., 2005; Donaldson et al., 2001; Fiez, 1997; Friederici et al., 2000; Petersen et al., 1988; Roskies et al., 2001; Thompson-Schill et al., 1997; Wagner et al., 2001) would have differences as a function of the type of stimulus (word vs. PW) being processed.

\section{Methods:}

Participants

The original study sample consisted of 28 participants; after excluding participants who had incomplete or compromised data quality, our study included 24 individuals. Participants were 24 monolingual (English-speaking), right-handed participants (12 male, mean age 24.8 years, 2.57 std. dev) recruited from neighborhoods surrounding Washington University in Saint Louis as well as from the university student body (Nardos, 2015). All participants had no history of psychiatric or neurological illness and scored above the $50^{\text {th }}$ percentile on the WoodcockJohnson III reading assessment (Woodcock \& Johnson, 2002). The Washington University Human Studies Committee approved the study (IRB ID \# 201202083) and all participants were reimbursed for their participation.

$\underline{\text { Task }}$

In a visually-presented lexical decision task, individuals identified words vs. PWs while in the MRI scanner via button pressing. A set of words (50\% animals; 50\% artifacts; 3-9 letters; 1-3 syllables) and PWs (5 letters, 1 or 2 syllables) were selected from the English Lexicon Project (Balota et al., 2007; Nardos, 2015). When in the scanner participants had two buttons, one on each hand. Each button corresponded either to words or PWs, participants pressed the buttons with the thumb of either hand to identify the stimuli. Stimuli were presented in a widely spaced manner, i.e. separated by $\sim 20$ s, to avoid hemodynamic response signal overlap across individual stimuli and allow extraction of individual trial BOLD responses (Nardos, 2015). In a given trial, a word or PW stimulus was presented for 2.5 seconds (1 TR or MR frame) with each letter subtending $0.5^{\circ}$ of horizontal visual angle, followed by 17.5 seconds (7TRs or MR frames) of a black fixation screen with a white cross. Participant underwent 10 functional MRI runs each with 24 stimuli (18 PWs and 6 words) per run. Communication with participants was facilitated by MR-compatible headphones which were also used to reduce noise from the scanner. Head movement was minimized by using a molded thermoplastic mask. Stimuli were presented using Psyscope (Cohen, MacWhinney, Flatt, \& Provost, 1993) installed on an iMAC computer (Apple, Cupertino, CA) and projected via an LCD projector (Sharp model PG-C20XU) onto an MRIcompatible rear-projection screen combined with a mirror attached to the head coil (CinePlex).

\section{Data acquisition}

Structural and functional MRI data were collected as described in Nardos, 2015 (Nardos, 2015) from a Siemens 3 Tesla MAGNETOM Trio system (Erlangen, Germany). The scanner included total imaging matrix technology (TIM) and utilized a 12-channel head matrix coil. A high resolution a T1-weighted MP-RAGE was acquired $(\mathrm{TE}=3.08 \mathrm{~ms}$, TR [partition] $=2.4 \mathrm{~s}, \mathrm{TI}=$ $1000 \mathrm{~ms}$, flip angle = 8", 176 slices with 1 X 1 X 1 mm voxels). To improve atlas alignment a 
T2-weighted turbo spin echo structural image $(\mathrm{TE}=84 \mathrm{~ms}, \mathrm{TR}=6.8 \mathrm{~s}, 32$ slices with $2 \mathrm{X} 1 \mathrm{X}$ $4 \mathrm{~mm}$ voxels) matching the acquisition plane of the BOLD images were also collected. Alignment to the anterior commissure-posterior commissure (AC-PC) plane was performed by Siemens pulse sequence protocol. BOLD contrast-sensitive gradient echo echo-planar sequence $(\mathrm{TE}=27 \mathrm{~ms}$, flip angle $=90 "$, in-plane resolution $=4 \mathrm{X} 4 \mathrm{~mm})$ was used for functional data collection. Using a TR of 2.5 seconds, 32 contiguous, $4 \mathrm{~mm}$ - thick axial slices whole-brain EPI volumes were collected. To allow steady state magnetization, the first four volumes of each run were discarded.

\section{$\underline{\text { fMRI data preprocessing }}$}

Data were processed using surface-based registration applying a modified version from the Human Connectome Project (Glasser et al., 2013) plus in-house denoising methods (https://github.com/DCAN-Labs). Processing includes the use of FSL (Jenkinson, Beckmann, Behrens, Woolrich, \& Smith, 2012; Smith et al., 2004; Woolrich et al., 2009) and FreeSurfer tools (Desikan et al., 2006; Fischl \& Dale, 2000; Sereno et al., 1995). Briefly, gradient distortion corrected T1-weighted and T2-weighted volumes were first aligned to the MNI's AC-PC axis and then non-linearly normalized to the MNI atlas. Later, the T1w and T2w volumes were reregistered using boundary-based registration (Greve \& Fischl, 2009) to improve alignment. The BOLD data were corrected for field distortions (using FSL's TOPUP) and processed by doing a preliminary 6 degrees of freedom linear registration to the first frame. After this initial alignment, the average frame was calculated and used as a final reference. Next, the BOLD data were registered to this final reference and to the T1-weighted volume, all in one single step, by concatenating all the individual registrations into a single registration. Individual brains were segmented using recon-all from FreeSurfer. Segmentations were improved by using the enhanced white matter-pial surface contrast of the T2-weighted sequence. Additionally, the initial pial and white matter surfaces were used to distinguish an initial cortical ribbon. From these segmentations, a tailored 3D surface was created for each participant and registered to the Conte 69 surface atlas of the Human Connectome Project.

The cortical ribbon defined by the structural T1-weighted and T2-weighted volumes was used to define a high-resolution mesh used for surface registration of the BOLD data. This cortical ribbon was also used to quantify the partial contribution of each voxel in the BOLD data in surface registration. Timecourses in the cortical mesh were calculated by obtaining the weighted average of the voxels neighboring each vertex within the grid, where the weights are given by the average number of voxels wholly or partially within the cortical ribbon. Voxels with a high coefficient of variation, indicating difficulty with tissue assignment or containing large blood vessels, were excluded. Next, the resulting timecourses in this mesh were downsampled into a standard space of 91, 282 anchor points (grayordinates), which were defined in the brain atlas and mapped uniquely to each participant's brain after smoothing them with a $2 \mathrm{~mm}$ full-widthhalf-max Gaussian filter. Subcortical regions were treated and registered as volumes. Two-thirds of the grayordinates were vertices located in the cortical ribbon while the remaining grayordinates were subcortical voxels. Subsequently, resulting timecourses (surface registration for cortex and volume registration for subcortical gray matter) were detrended and further processed to remove the effect of movement such that, volumes where the total relative movement in any direction (frame displacement, FD) in relation to the previous volume were 
greater than $0.2 \mathrm{~mm}$ were censored (Power, Barnes, Snyder, Schlaggar, \& Petersen, 2012; Siegel et al., 2013).

$\underline{\text { ROIs and functional networks }}$

Collected BOLD data were parcellated using the Gordon schema with 12 networks composed of 333 regions of interest (ROIs). Each grayordinate was assigned a network and region within this parcellation. The networks, their abbreviation and the number of ROIs included are: Auditory (Aud, n=24), Cingulo Opercular ( $\mathrm{CiO}, \mathrm{n}=40)$, Cingulo Parietal (CiP, n=5), Default (Def, $n=41$ ), Dorsal Attention (DoA, n=32), Fronto-Parietal (FrP, n=24), Retrosplenial Temporal (ReT, n=8), Somato-sensory hand (Sml, $\mathrm{n}=38)$, Somato-sensory mouth $(\mathrm{SMm}, \mathrm{n}=8)$, Salience ( $\mathrm{Sal}, \mathrm{n}=4)$, Ventral Attention (VeA, n=23), and Visual (Vis, n=39). From the 333 ROIs, 47 ROIs were not assigned to any network. The functional networks are visually described in Figure 1.

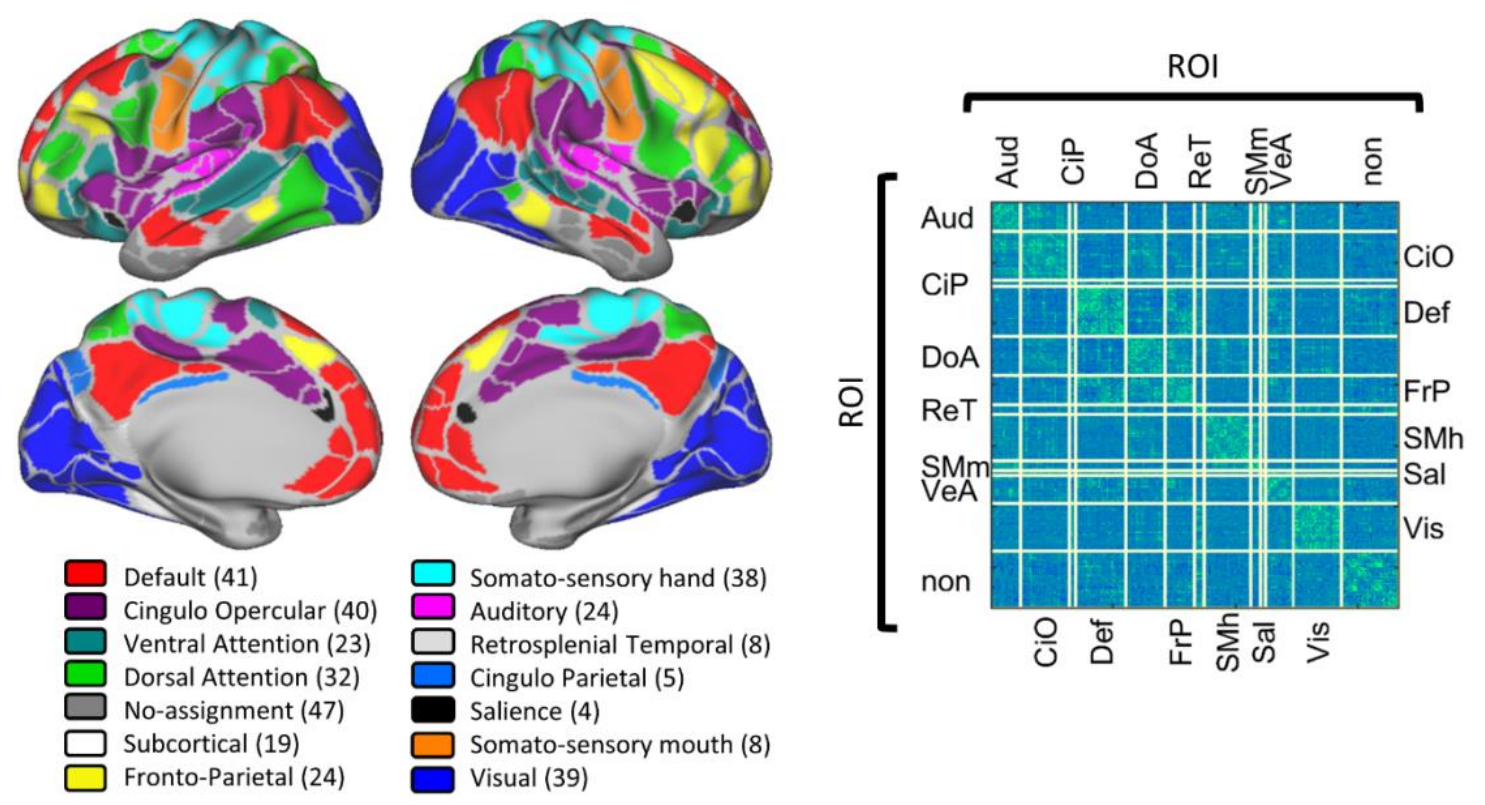

Figure 1: The Gordon parcellation schema was used to separate BOLD data into functional networks.

The left side displays a visual representation of the 13 defined cortical networks in the Gordon Parcellation. Network are color codes with the number of ROIs included in parentheses (this parcellation defines cortical areas into 333 ROIs, 47 of which are not assigned to any network)

On the right is a model connectivity matrix, displaying how connectivity between ROIs was organized using the 13 networks defined on the left panel.

\section{Motion censoring}

Correction for head motion was completed by calculating 6 parameters of head movement, movement and rotation along the $\mathrm{x}, \mathrm{y}$, and $\mathrm{z}$ axes. The absolute sum of movement along these parameters was evaluated after each change of frame and termed the frame displacement (FD). For our study, we set our FD threshold at $0.3 \mathrm{~mm}$ and set the FD of the first frame at 0 . This measure was only used as a way to detect motion and was not used for regression (Power et al., 2012). 
Grouping data for connectotyping

Connectotypes (model-based connectivity matrices) were calculated per participant at each frame and condition, i.e. for words and PWs, as shown in Figure 2, panel c. To do this we grouped the frames per TR (i.e., TR, from 1 to 8) and stimulus type (i.e., word and PW), ending up with 16 stacks of frames for each case (See Figure 2, panel c). We ensured that for consideration, a particular trial had to constitute 8 frames of data, with the additional constraint that the preceding trial in the experiment took place at least 20 seconds prior, ensuring that the timecourse for the current trial under consideration is not adulterated by that previous trial. Frames were excluded if head movement was higher than a given frame displacement (FD) threshold of $0.3 \mathrm{~mm}$ (Power, Jonathan D Anish Mitraa, Timothy O Laumanna, Abraham Z Snydera, b, Bradley L Schlaggar, 2014). 15 participants remained after motion censoring. Connectotypes were calculated if all the 16 cases had at least 40 frames. Connectotypes were calculated using the same number of frames on each condition. Such frames were selected randomly from the surviving frames with head movement lower than the pre-selected threshold. Data used to create individual connectotypes did not account for correct word or PW identification. 


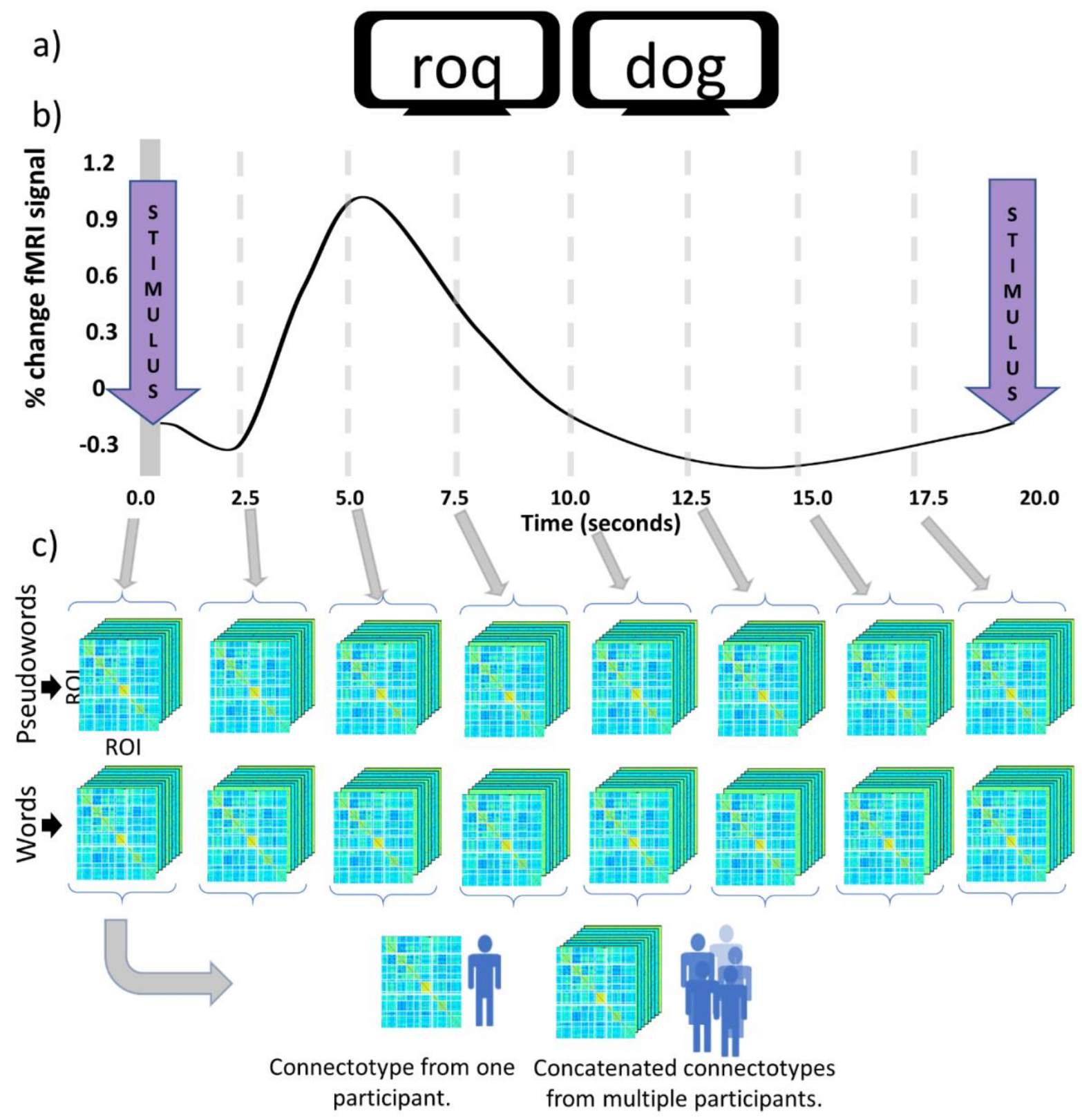

Figure 2: The widely-spaced design of the word vs PW experiment allows us to characterize dynamic changes in functional connectivity using connectotyping.

a) Example of what stimuli would look like in the scanner on the left is PW and on the right is a word.

b) The widely-spaced design of the data we used allowed us to present a stimulus every 20 seconds and collect whole brain data every 2.5 seconds. This allowed us to have 8 frames of data which are shown with dashed grey-lines.

c) For each participant, we separated the data by frame and stimuli type and then applied our connectotyping technique. We then grouped concatenated the connectotypes for all participants to perform statistical analyses testing for changes across frame and stimuli type. 


\section{Connectotyping}

As described in the original connectotyping publication, this approach calculates model-based connectivity matrices applying a linear model to the timecourses (Miranda-Dominguez, Mills, Carpenter, Grant, \& Kroenke, 2014). In our experiment connectotyping procedures (MirandaDominguez et al., 2014) were applied to the data of each participant separated by frame of task evolution and paradigm to characterize regional connectivity occurring during the evolution of a task. This created 16 connectotypes per participant: one per frame (1 to 8$)$ and task (word/PW) using the parcellated timecourses as defined by Gordon. Connectotyping created matricial connectomes with the dimension ROI X ROI (333 X 333) holding weighted values termed betaweights. These values represent the directional contribution of the signal from each brain area to another's signal representing each region's signal as a weighted sum of remaining brain regions, thereby they also account for all the connections occurring in the brain. Overfitting of connectomes was reduced by truncated singular value decomposition and removal of autocorrelation was avoided because of interference with collected functional data (MirandaDominguez, Feczko, Grayson, Walum, Nigg, \& Fair, 2018a).

\section{Statistical analysis}

Independent repeated measures ANOVA tests were run for each functional system's pair using MATLAB. For this pilot study, we excluded primary somatosensory and unimodal networks and included only the following functional systems: Cingulo Parietal, Default, Dorsal Attention, Fronto-Parietal, Salience, and Ventral Attention, ending up with 36 functional system's pairs. Table 1 shows all the functional system pairs including the count of unique beta-weights. The 2way repeated measures ANOVA used each participant's beta weights grouped two withinsubject factors, i.e. frame (1 to 8 ), stimulus type, i.e. (word/PW) and their interaction. In MATLAB, the repeated measures ANOVA tests are performed in two steps. First, a linear mixed effects model was fit to predict optimal beta weights as a function of frame, stimulus type and the interaction between the two within-subject factors. The resulting marginal means (betaweighted values) are grouped according to the said factors to characterize statistical differences (See Figure 3 for a visualization of the distribution of the marginal means of the data functional system's pairs included in this study). A false discovery rate was used as a correction for the multiple comparisons across networks, and if Mauchly's Test for asymmetry was not met epsilon adjusted values were used. When indicated, connectivity data was box-cox transformed to normalize distributions (Montgomery, 2005) and the logarithmic base was optimized by gradient descent. Differences were assessed as significant using a threshold of 0.05. All statistical analyses were performed in MATLAB. 
Table 1. Grouping of connections per functional system pair.

\begin{tabular}{|c|c|c|}
\hline Count & Functional system pair & Number of connections \\
\hline 1 & $\mathrm{CiP}$ and $\mathrm{CiP}$ & 20 \\
\hline 2 & Def and CiP & 205 \\
\hline 3 & DoA and CiP & 160 \\
\hline 4 & $\mathrm{FrP}$ and $\mathrm{CiP}$ & 120 \\
\hline 5 & Sal and CiP & 20 \\
\hline 6 & VeA and CiP & 115 \\
\hline 7 & $\mathrm{CiP}$ and Def & 205 \\
\hline 8 & Def and Def & 1640 \\
\hline 9 & DoA and Def & 1312 \\
\hline 10 & FrP and Def & 984 \\
\hline 11 & Sal and Def & 164 \\
\hline 12 & VeA and Def & 943 \\
\hline 13 & CiP and DoA & 160 \\
\hline 14 & Def and DoA & 1312 \\
\hline 15 & DoA and DoA & 992 \\
\hline 16 & FrP and DoA & 768 \\
\hline 17 & Sal and DoA & 128 \\
\hline 18 & VeA and DoA & 736 \\
\hline 19 & $\mathrm{CiP}$ and $\mathrm{FrP}$ & 120 \\
\hline 20 & Def and FrP & 984 \\
\hline 21 & DoA and $\mathrm{FrP}$ & 768 \\
\hline 22 & $\mathrm{FrP}$ and $\mathrm{FrP}$ & 552 \\
\hline 23 & Sal and FrP & 96 \\
\hline 24 & VeA and FrP & 552 \\
\hline 25 & CiP and Sal & 20 \\
\hline 26 & Def and Sal & 164 \\
\hline 27 & DoA and Sal & 128 \\
\hline 28 & FrP and Sal & 96 \\
\hline 29 & Sal and Sal & 12 \\
\hline 30 & VeA and Sal & 92 \\
\hline 31 & $\mathrm{CiP}$ and $\mathrm{VeA}$ & 115 \\
\hline 32 & Def and VeA & 943 \\
\hline 33 & DoA and $V e A$ & 736 \\
\hline 34 & FrP and VeA & 552 \\
\hline 35 & Sal and VeA & 92 \\
\hline 36 & VeA and VeA & 506 \\
\hline
\end{tabular}

Table 1: List of all 36 functional system pairs tested in the ANOVA with the number of ROI connections between networks listed in the third column.

\section{Results:}

Repeated measures ANOVA were performed for each possible functional system pairs among six networks of interest, the Cingulo Parietal, Default, Dorsal Attention, Fronto-Parietal, Salience, and Ventral Attention networks from the Gordon parcellation (Gordon et al., 2014). A total of 36 functional system pairs were tested. The ANOVA examined activation differences across frame, stimulus type, and the interaction between the two factors, for each network pair. Our main results were derived from analysis of connectomes created from functional MRI data of 15 participants using 40 frames having FD values less than $0.3 \mathrm{~mm}$ at each frame and with 6 networks tested in the repeated measures ANOVA. The results from all of the functional system pairs tested are shown in Figure 3. 


\section{Network level connectivity differences across time and stimulus}

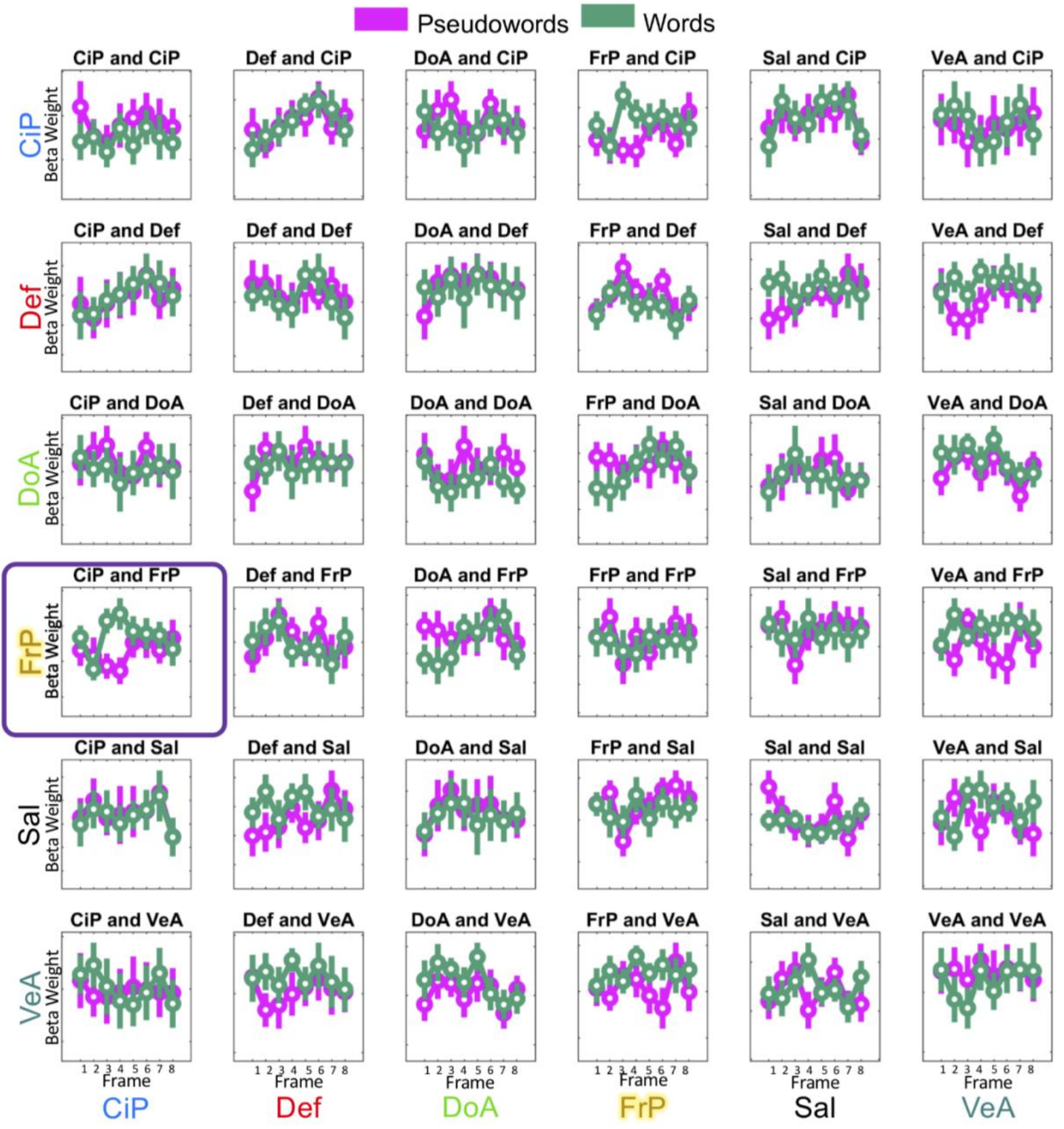

Figure 3: Results from repeated measures ANOVA comparing network level connectivity differences using connectotyping.

To establish network level differences, the beta-weights representing the connectivity between each network to the remaining networks were plotted. The functional system pairs tested were between the following six networks: Auditory (Aud, n=24), Cingulo Opercular (CiO, n=40), Cingulo Parietal (CiP, n=5), Default (Def, n=41), Dorsal Attention (DoA, n=32), Fronto-Parietal (FrP, n=24), Retrosplenial Temporal (ReT, n=8), Somato-sensory hand (Sml, $\mathrm{n}=38)$, Somato-sensory mouth $(\mathrm{SMm}, \mathrm{n}=8)$, Salience (Sal, $\mathrm{n}=4$ ), Ventral Attention (VeA, $n=23$ ), and Visual (Vis, $n=39$ ) networks. 36 separate 
functional system pairs were tested. For each comparison of how the network listed on the leftmost side predicts the network listed at the bottom of the figure, individual functional system pair plots were created. The y-axis of each pair represents the beta weight prediction between the networks. The center circle represents the mean and the error bars show 1.15 standard deviations. The frame was plotted on the $\mathrm{x}$-axis of each plot. These plots show the beta-weight for both word (green) and PW (purple) stimuli. From this analysis we found a significant difference in how the Fronto-Parietal and Cingulo Parietal networks communicate over time and per stimuli type, this finding is outlined in purple.

\section{Differences in frame}

When comparing differences across time, we found significant differences across frames $(\mathrm{F}=3.7330, p=0.046$, corrected) for beta-weights relating the Fronto-Parietal and Default networks, as shown in Figure 4. Differences were driven by changes in beta weights between frames 3 and 7(t-test, $\mathrm{p}=0.018$ ), frames 1 and 3 (t-test, $\mathrm{p}=0.041$ ), and frames 1 and 6 (t-test, $\mathrm{p}=0.043)$.

\section{Frontal-Parietal and Default temporal connectivity comparison}

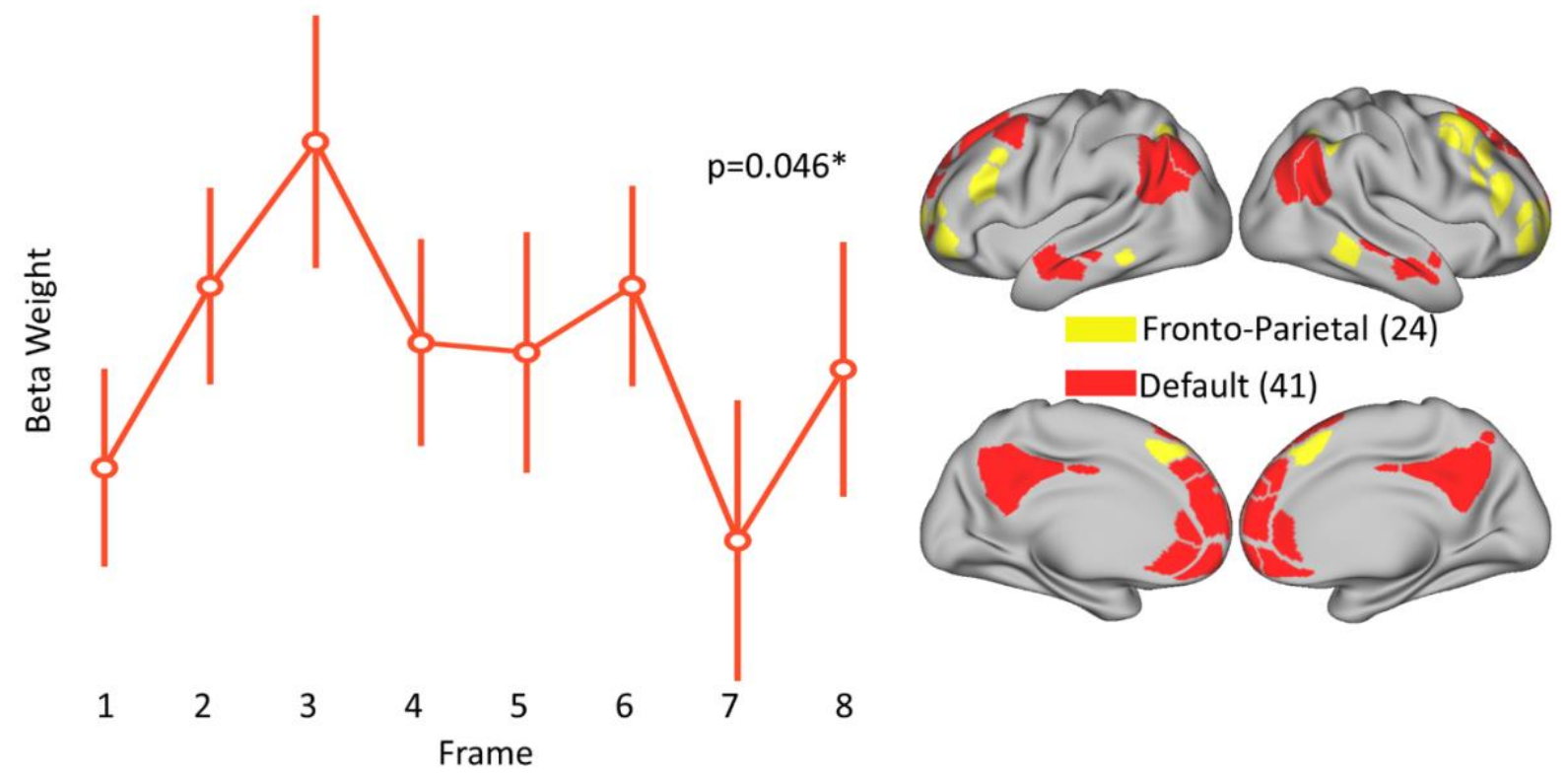

Figure 4: Differences across frames of task progression found to be significant between the Fronto-Parietal and Default networks.

The left panel shows the change in beta-weights between the Default and Fronto-Parietal networks. Changes were found to be significant with a corrected p-value of 0.046 .

The right panel shows a topological representation of cortical areas for both the FrontoParietal (yellow) and Default (red) networks. 
Differences across stimulus types (word/PW)

We found no significant network level connectivity differences based on word or non-word exposure. The greatest differences in network-network connectivity were found within the Dorsal Attention; between the Cingulo Parietal and Fronto-Parietal; and between the FrontoParietal and Cingulo Parietal networks. These three functional system pairs had network-network differences with corrected p-values of 0.117 .

Differences for the interaction

We found significant differences in beta weights for the interaction of frame and task for the Cingulo Parietal and Fronto-Parietal systems ( $\mathrm{F}=3.7155, \mathrm{p}=0.047$, corrected). Differences were driven by the dynamic changes of beta-weights at frames 3 (paired t-test word vs non-word, $\mathrm{p}=2.65 \mathrm{e}-5$ ) and 4 (paired t-test word vs non-word, $\mathrm{p}=2.68 \mathrm{e}-4$ ), as shown in Figure 5.

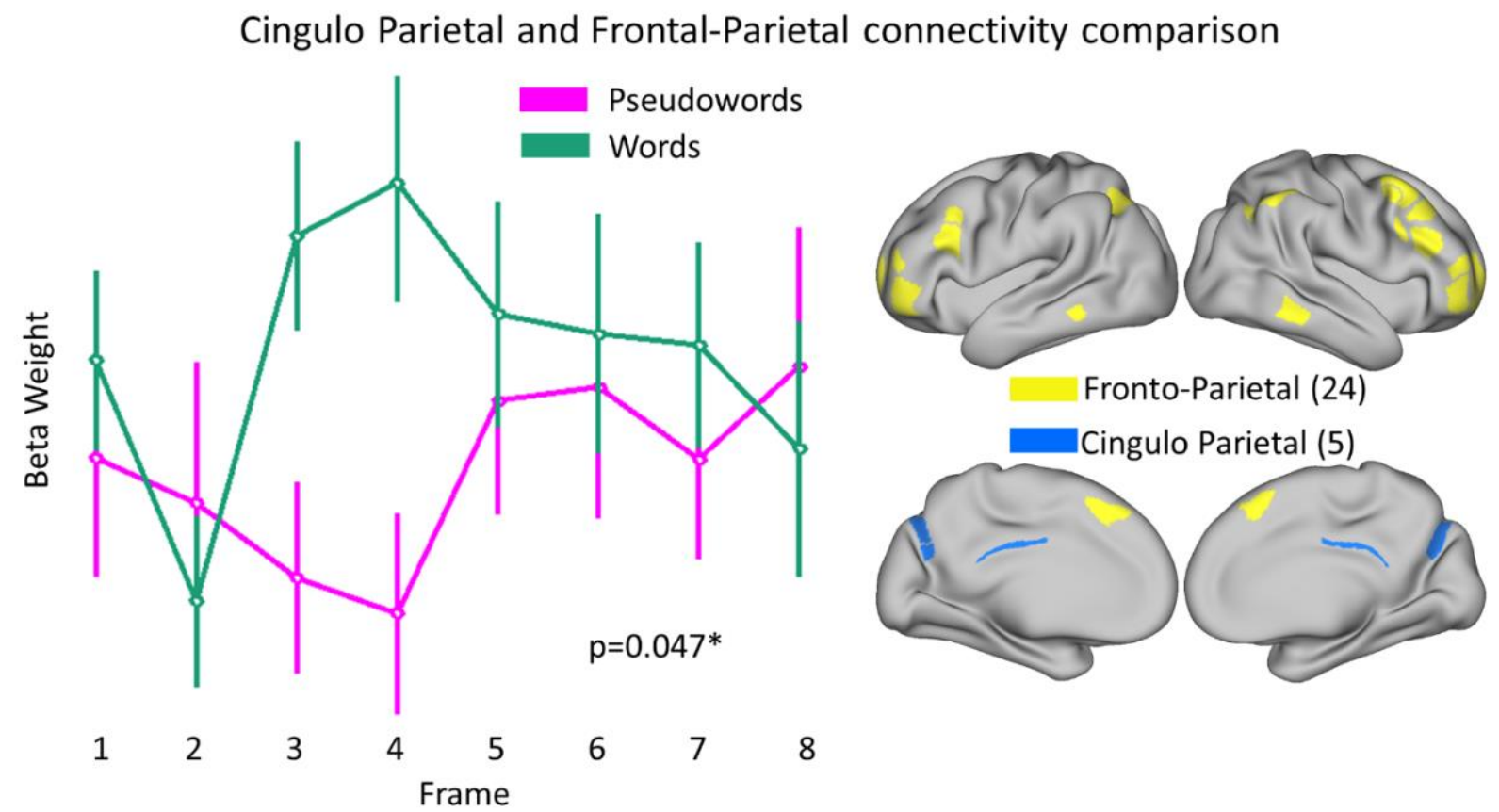

Figure 5: Significant difference observed in how the Cingulo Parietal and Fronto-Parietal networks interact due to both progression of task and stimulus type.

The left panel shows the change in beta-weights between the Fronto-Parietal and Cingulo Parietal networks. When testing for how these values changed across frame and stimuli type, this functional system pair was found to be significant with a corrected $\mathrm{p}$-value of 0.047. Beta weights represent how the Fronto-Parietal network interacts with the Cingulo Parietal network.

The right panel shows the topological representation of cortical areas for both the Fronto-Parietal (yellow) and Cingulo Parietal (blue) networks.

Robustness of results at different motion censoring thresholds

To test the robustness of our analysis using a more stringent threshold, we calculated connectotypes using an FD of 0.25 and recalculated the connectotypes and corresponding repeated measures ANOVA tests. While results were no significant, the Cingulo Parietal and 
Fronto-Parietal systems also exhibited the strongest differences in beta-weights for the interaction of frame and stimulus type ( $\mathrm{p}=0.0698$, corrected), as shown in Figure 5, Supplement 1. In addition, we also recalculated our analysis with an FD of 0.5 , allowing for more participants and found a similar beta-weight response between these two networks. However, when correcting for multiple comparisons, the findings were not significant (Figure 5, Supplement 1). These similarities in observed connectivity between the Cingulo Parietal and Fronto-Parietal networks at different thresholds display a trend in temporal connectivity response.

Characterizing changes in connectivity values in connectivity matrices calculated using Pearsoncorrelations

We repeated all the previous analysis using connectivity matrices calculated via Pearson correlations instead of connectotypes using the same frames used to calculate connectotype. No FD threshold led to significant differences in functional connectivity. Figure 5 supplement 2 shows the distribution of marginal means of connectivity values when connectivity matrices were calculated using an FD threshold of 0.3 (i.e., the same threshold used for connectotyping).

\section{Discussion:}

Connectopying allows for characterization of dynamic functional connectivity changes during task performance

Recent advances in rs-fcMRI analysis approaches have led to increased understanding of brain functioning - where experimental designs have been able to identify brain areas supporting consciousness (Lloyd, 2002), moral judgment (Greene, Sommerville, Nystrom, Darley, \& Cohen, 2001), as well as heritable patterns of brain connectivity (Miranda-Dominguez, Feczko, Grayson, Walum, Nigg, \& Fair, 2018). Successful execution of mental tasks might require the collaboration of different brain systems in a timely manner. Activation dynamics occurring during overt cognitive tasks have yet to be explored. Given the noisy nature of functional MRI and the averages that are typically done to improve signal to noise ratios, a smother signal comes at the price of blurring individual and dynamic changes in functional connectivity. In this study, we aimed to track temporal changes in brain connectivity during task performance at the individual subject level using connectotyping, an efficient way to calculate dynamic functional connectivity between brain regions. We tested the viability of connectotyping on task data from a lexical decision-based fMRI study that used a widely spaced event-related design ( 20 second trials). The use of this particular dataset allowed for the hemodynamic response function corresponding to a single stimulus to be detected without signal interference from the next or preceding stimulus. Our approach has the potential to reveal how functional connections between ROIs (here at the network level) progress during the performance of a task not just at the peak of activation. As hypothesized, application of connectotyping to the word vs PW dataset revealed significant dynamic (i.e. across frames) connectivity differences between the Cingulo Parietal and Fronto-Parietal networks, as a function of stimulus type (i.e. word vs. PW). Our interpretation of these findings is further elaborated below.

The significantly different dynamic temporal relation occurring as a function of stimulus type between the Cingulo Parietal and Frontal-Parietal networks suggest that the evolving and directional contributions from the Fronto-Parietal to the Cingulo Parietal network are distinct in pattern depending on whether participants were viewing something meaningful (i.e. word) vs. 
meaningless (i.e. PW). Additionally, because connectotypes are calculated through beta-weights which display how each region of the brain interacts with another our results can state which network is driving these differences. Prior studies using correlations have not been able to identify the direction of information flow for task performance and has limited analyses. Through our model we are able to identify changes in the way one network influences another, providing directional resolution to the connectivity between networks.

After further testing and creating connectotypes with more and less stringent movement thresholds (at 0.25 and 0.5 frame displacement thresholds), this observed Cingulo Parietal and Frontal-Parietal network pattern of coactivation persisted, implying the stability of the findings (Figure 5 supplement 1). These trends, however, did not survive corrections for multiple comparisons. Although these additional analyses did not withstand statistical significance, the presence of the same pattern of results supports the robustness of our primary finding.

The presence of dynamic connectivity differences between the Cingulo Parietal and FrontoParietal networks support our principal hypothesis that task dependent regional brain communication changes during task progression; something that to our knowledge is a novel finding. Our findings consequently also validate the use of connectotyping as a tool for task fMRI analysis which can provide a novel depiction of brain activity including dynamic temporal changes in functional connectivity.

Although our current methodological approach is notably different from prior traditional task fMRI analysis approaches, we did expect some overlap in the identified functional neuroanatomy involved in distinguishing word vs. PW. Exposure to words vs. PWs resulted in significantly different temporal connectivity patterns between areas known to have a role in cognitive control, semantic processing, and memory retrieval. The Fronto-Parietal network is characterized as a task control network that has a particular role in the adaptive moment-to-moment requirements of a cognitive task such as task instantiation and dynamic feedback or error detection (Dosenbach et al., 2007). In 2013, Cole et al. produced evidence suggesting that the FrontoParietal network works as a cognitive hub by communicating with other control and processing networks to allow cognitive adaption during tasks (Cole et al., 2013). The Fronto-Parietal network also initiates and adjusts cognitive control to produce higher-level cognitive functions (Marek \& Dosenbach, 2018). Here, the fact that such an adaptive control network displays distinct relations as a function of stimulus type is consistent with an expectation that resolution of the identity of a word vs. nonword may have different cognitive control demands.

The regions corresponding to the Cingulo Parietal network have previously been linked with memory retrieval processes (Power et al., 2011). Parts of the Cingulo Parietal network are found in the precuneus and near the posterior cingulate, regions that have previously been linked with semantic processing. For instance, the regions have been shown to distinguish between words and PWs in prior work using traditional fMRI analysis (Binder, Desai, Graves, \& Conant, 2009). The same two regions have also previously been associated with supporting word learning in young adults (Nardos, 2015). In addition, there is ample prior work that has associated those same two regions with memory retrieval (Cabeza et al., 2008; Dobbins \& Wagner, 2005; Nelson et al., 2010; Rugg \& Vilberg, 2013; Wheeler \& Buckner, 2003; Yonelinas \& Levy, 2002; Yonelinas et al., 2005). In aggregate, the aforementioned findings linking regions in the Cingulo 
Parietal network with semantic processing and memory retrieval is consistent with our finding that dynamic functional connectivity between this network and the Fronto-Parietal network supporting adaptive cognitive control is what distinguishes meaningful words from meaningless PWs.

Connectotyping provides additional understanding of brain activity

Our study not only describes the presence of a connectivity pattern that occurs between two networks but shows that this connectivity evolves throughout the progression of the task. We not only identify networks key to the differential processing of words vs. PWs but also display that these networks are tuning their level of communication with each other in a dynamic manner across time. Thus, our results show that this functional system pair can be influenced by the type of lexical stimuli and that these observed changes in this connectivity likely reflect differences in the interactive functional neuroanatomy recruited in processing meaningful vs. meaningless stimuli. While our findings are supported both by repeated testing and established functions of the Cingulo Parietal and Fronto-Parietal networks, there is no current precedent for the validity of tracking temporal connectivity changes on task data.

These results also provide the direction of connectivity flow through the beta weights used to model activation patterns, in our finding the Fronto-Parietal network is changing how it interacts with the Cingulo-Parietal network to produce these overall network level effects shown in Figure 5 which we believe indicate differential processing methods for our stimuli. Because the FrontoParietal network's established role in modulating tasks through moment-to-moment control and dynamic feedback, this directionality which our findings have is further supported. For this task it is possible that the Frontal-Parietal network exerted through moment-to-moment control and dynamic feedback to the Cingulo Parietal network to drive observed differences, supporting the directionality of our findings.

Limitations and future work

Because of our stringent motion censoring, our analyses are based on the data of only 15 participants of a narrow age range, which could limit the generalizability of our results. In this exploratory study, we decided to focus on higher order heterometal systems and to exclude primary sensory cortex. Studies with a larger number of participants and different tasks might allow the inclusion of more systems. The usage of a widely-spaced dataset was ideal to test the feasibility of using connectotyping to track dynamic changes in functional connectivity. A widely-spaced design, however, limits the number of contrasts that can be performed and measured. As we succeed in using a linear model to track dynamic changes, superposition and convolution can be used in event-related experiments where stimuli can be changed at each TR. By applying those validated methods to deconvolve the beta weights corresponding to each frame and stimulus, the same statistical analysis (i.e., repeated measures ANOVA) can be used to track dynamic changes in functional connectivity.

While limited by the constraints of our data and the novelty of our approach, our findings serve to expand on the roles and functions of the Cingulo Parietal and Fronto-Parietal networks as an incentive for others to pursue analyses which account for patterns of dynamic whole-brain connectivity and provide temporal resolution. The application of this approach to additional studies exploring other tasks and with differentially spaced study designs will not only further 
validate the use of this approach but also has the potential to expand our understanding of brain activity during the performance of a task.

\section{References:}

Badre, D., Poldrack, R. A., Paré-Blagoev, E. J., Insler, R. Z., \& Wagner, A. D. (2005). Dissociable Controlled Retrieval and Generalized Selection Mechanisms in Ventrolateral Prefrontal Cortex. Neuron, 47(6), 907-918. https://doi.org/10.1016/j.neuron.2005.07.023

Balota, D. A., Yap, M. J., Cortese, M. J., Kessler, B., Loftis, B., Neely, J. H., ... Treiman, R. (2007). The English lexicon project. Behavioral Research Methods, 39(3), 445-459. https://doi.org/10.3758/BF03193014

Binder, J. R., Desai, R. H., Graves, W. W., \& Conant, L. (2009). Where Is the Semantic System? A Critical Review and Meta-Analysis of 120 Functional Neuroimaging Studies, (December). https://doi.org/10.1093/cercor/bhp055

Biswal, B., Zerrin Yetkin, F., Haughton, V. M., \& Hyde, J. S. (1995). Functional connectivity in the motor cortex of resting human brain using echo-planar mri. Magnetic Resonance in Medicine, 34(4), 537-541. https://doi.org/10.1002/mrm.1910340409

Botvinick Todd S Braver, M. M., Barch, D. M., Carter, C. S., Cohen, J. D., Botvinick, M. M., \& Braver, T. S. (2001). Conflict Monitoring and Cognitive Control. Cohen \& ServanSchreiber (Vol. 108). Posner \& Petersen. Retrieved from https://pdfs.semanticscholar.org/05b1/99fc0e63bc3a7975f9112b79fa0d21bd3161.pdf

Braver, T. S., \& Barch, D. M. (2006). Extracting core components of cognitive control. Trends in Cognitive Sciences, 10(12), 529-532. https://doi.org/10.1016/j.tics.2006.10.006

Cabeza, R., Ciaramelli, E., Olson, I. R., \& Moscovitch, M. (2008). Parietal Cortex and Episodic Memory: An Attentional Account. Nature Reviews Neuroscience, 9(8), 613-625. https://doi.org/10.1038/nrn2459.Parietal

Cohen, J. D., MacWhinney, B., Flatt, M., \& Provost, J. (1993). PsyScope: A new graphic interactive environment for designing psychology experiments. Behavioral Research Methods, Instruments, and Computers, 25(2), 257-271.

Cole, M. W., Reynolds, J. R., Power, J. D., Repovs, G., Anticevic, A., \& Braver, T. S. (2013). Multi-task connectivity reveals flexible hubs for adaptive task control. Nature Neuroscience, 16, 1348. Retrieved from https://doi.org/10.1038/nn.3470

Desikan, R. S., Ségonne, F., Fischl, B., Quinn, B. T., Dickerson, B. C., Blacker, D., ... Killiany, R. J. (2006). An automated labeling system for subdividing the human cerebral cortex on MRI scans into gyral based regions of interest. NeuroImage, 31(3), 968-980. https://doi.org/10.1016/j.neuroimage.2006.01.021

Dobbins, I. G., \& Wagner, A. D. (2005). Domain-general and Domain-sensitive Prefrontal Mechanisms for Recollecting Events and Detecting Novelty. Cerebral Cortex, 15(11), 1768-1778. https://doi.org/10.1093/cercor/bhi054

Donaldson, D. I., Petersen, S. E., Ollinger, J. M., \& Buckner, R. L. (2001). Dissociating State and Item Components of Recognition Memory Using fMRI. NeuroImage, 13(1), 129-142. https://doi.org/10.1006/NIMG.2000.0664

Dosenbach, N. U. F., Fair, D. A., Miezin, F. M., Cohen, A. L., Wenger, K. K., Dosenbach, R. A. T., ... Petersen, S. E. (2007). Distinct brain networks for adaptive and stable task control in humans, 104(26), 11073-11078.

Dosenbach, N. U. F., Visscher, K. M., Palmer, E. D., Miezin, F. M., Wenger, K. K., Kang, H. C., 
... Petersen, S. E. (2006). A Core System for the Implementation of Task Sets, 799-812. https://doi.org/10.1016/j.neuron.2006.04.031

Engel, S. A., Rumelhart, D. E., Wandell, B. A., Lee, A. T., Glover, G. H., Chichilnisky, E.-J., \& Shadlen, M. N. (1994). fMRI of human visual cortex. Nature, 369(6481), 525-525. https://doi.org/10.1038/369525a0

Fiez, J. A. (1997). Phonology, semantics, and the role of the left inferior prefrontal cortex. Human Brain Mapping, 5(2), 79-83. https://doi.org/10.1002/(SICI)10970193(1997)5:2<79::AID-HBM1>3.0.CO;2-J

Fischl, B., \& Dale, A. M. (2000). Measuring the thickness of the human cerebral cortex from magnetic resonance images. Proceedings of the National Academy of Sciences of the United States of America, 97(20), 11050-11055.

https://doi.org/https://doi.org/10.1073/pnas.200033797

Friederici, A. D., Opitz, B., \& von Cramon, D. Y. (2000). Segregating Semantic and Syntactic Aspects of Processing in the Human Brain: an fMRI Investigation of Different Word Types. Cerebral Cortex, 10(7), 698-705. https://doi.org/10.1093/cercor/10.7.698

Glasser, M. F., Sotiropoulos, S. N., Wilson, J. A., Coalson, T. S., Fischl, B., Andersson, J. L., ... WU-Minn HCP Consortium. (2013). The minimal preprocessing pipelines for the Human Connectome Project. NeuroImage, 80, 105-124. https://doi.org/10.1016/j.neuroimage.2013.04.127

Goebel, R., Khorram-Sefat, D., Muckli, L., Hacker, H., \& Singer, W. (2001). The constructive nature of vision: direct evidence from functional magnetic resonance imaging studies of apparent motion and motion imagery. European Journal of Neuroscience, 10(5), 15631573. https://doi.org/https://doi.org/10.1046/j.1460-9568.1998.00181.x

Gordon, E. M., Laumann, T. O., Adeyemo, B., Huckins, J. F., Kelley, W. M., \& Petersen, S. E. (2016). Generation and Evaluation of a Cortical Area Parcellation from Resting-State Correlations. Cerebral Cortex (New York, N.Y. : 1991), 26(1), 288-303. https://doi.org/10.1093/cercor/bhu239

Greene, J. D., Sommerville, R. B., Nystrom, L. E., Darley, J. M., \& Cohen, J. D. (2001). An fMRI investigation of emotional engagement in moral judgment. Science (New York, N.Y.), 293(5537), 2105-2108. https://doi.org/10.1126/science.1062872

Greve, D. N., \& Fischl, B. (2009). Accurate and robust brain image alignment using boundarybased registration. NeuroImage, 48(1), 63-72. https://doi.org/10.1016/j.neuroimage.2009.06.060

Iidaka, T., Matsumoto, A., \& Nogawa, J. (2006). Frontoparietal Network Involved in Successful Retrieval from Episodic Memory. Spatial and Temporal Analyses Using fMRI and ERP, (September). https://doi.org/10.1093/cercor/bhl040

Jenkinson, M., Beckmann, C. F., Behrens, T. E. J., Woolrich, M. W., \& Smith, S. M. (2012). FSL. NeuroImage, 62(2), 782-790. https://doi.org/10.1016/j.neuroimage.2011.09.015

Lloyd, D. (2002). Functional MRI and the Study of Human Consciousness. Journal of Cognitive Neuroscience, 14(6), 818-831. https://doi.org/10.1162/089892902760191027

Logothetis, N. K. (2003). The Underpinnings of the BOLD Functional Magnetic Resonance Imaging Signal, 23(10), 3963-3971.

Logothetis, N. K., Pauls, J., Augath, M., Trinath, T., \& Oeltermann, A. (2001). Neurophysiological investigation of the basis of the fMRI signal.

Marek, S., \& Dosenbach, N. U. F. (2018). The frontoparietal network: function, electrophysiology, and importance of individual precision mapping. Dialogues Clinical 
Neuroscience, 20(2), 133-140.

Miranda-Dominguez, O, Mills, B. D., Carpenter, S. D., Grant, K. A., \& Kroenke, C. D. (2014). Connectotyping: Model Based Fingerprinting of the Functional Connectome. PLoS ONE, 9(11), 111048. https://doi.org/10.1371/journal.pone.0111048

Miranda-Dominguez, Oscar, Feczko, E., Grayson, D. S., Walum, H., Nigg, J. T., \& Fair, D. A. (2018). Heritability of the human connectome: A connectotyping study. Network Neuroscience, 2(2), 175-199. https://doi.org/10.1162/netn_a_00029

Moerel, M., De Martino, F., \& Formisano, E. (2014). An anatomical and functional topography of human auditory cortical areas. Frontiers in Neuroscience, 8, 225. https://doi.org/10.3389/fnins.2014.00225

Montgomery, D. C. (Arizona S. U. (2005). Design and Analysis of Experiments, Sixth Edition. (J. Welter, T. Kulesa, \& P. McFadden, Eds.) (6th ed., Vol. 37). Hoboken, NJ: John Wiley \& Sons, Inc. https://doi.org/10.1080/00224065.2005.11980315

Nardos, B. (2015). Behavioral and $f$ MRI-based Characterization of Cognitive Processes Supporting Learning and Retrieval of Memory for Words in Young Adults. Retrieved from https://openscholarship.wustl.edu/cgi/viewcontent.cgi?article=1670\&context $=$ art_sci_etds

Nelson, S. M., Dosenbach, N. U. F., Cohen, A. L., Wheeler, M. E., Schlaggar, B. L., \& Petersen, S. E. (2010). Role of the anterior insula in task-level control and focal attention. Brain Structure \& Function, 214(5-6), 669-680. https://doi.org/10.1007/s00429-010-0260-2

Petersen, S. E., Fox, P. T., Posner, M. I., Mintun, M., \& Raichle, M. E. (1988). Positron emission tomographic studies of the cortical anatomy of single-word processing. Nature, 331(6157), 585-589. https://doi.org/10.1038/331585a0

Power, Jonathan D Anish Mitraa, Timothy O Laumanna, Abraham Z Snydera, b, Bradley L Schlaggar, S. E. P. (2014). Methods to detect, characterize, and remove motion artifact in resting state fMRI. Neuroimage, $84,1-45$. https://doi.org/10.1016/j.neuroimage.2013.08.048.Methods

Power, J. D., Barnes, K. A., Snyder, A. Z., Schlaggar, B. L., \& Petersen, S. E. (2012). Spurious but systematic correlations in functional connectivity MRI networks arise from subject motion. Neuroimage, 59(3), 2142-2154. https://doi.org/10.1016/j.neuroimage.2011.10.018.Spurious

Power, J. D., Cohen, A. L., Nelson, S. M., Wig, G. S., Barnes, K. A., Church, J. A., ... Petersen, S. E. (2011). Functional network organization of the human brain. Neuron, 72(4), 665-678. https://doi.org/doi:10.1016/j.neuron.2011.09.006

Roskies, A. L., Fiez, J. A., Balota, D. A., Raichle, M. E., \& Petersen, S. E. (2001). TaskDependent Modulation of Regions in the Left Inferior Frontal Cortex during Semantic Processing. Journal of Cognitive Neuroscience, 13(6), 829-843. https://doi.org/10.1162/08989290152541485

Rugg, M. D., \& Vilberg, K. L. (2013). Brain Networks Underlying Episodic Memory Retrieval Michael. Curr Opin Neurobiol., 23(2), 255-260.

https://doi.org/10.1016/j.conb.2012.11.005.

Sereno, M., Dale, A., Reppas, J., Kwong, K., Belliveau, J., Brady, T., ... Tootell, R. (1995). Borders of multiple visual areas in humans revealed by functional magnetic resonance imaging. Science, 268(5212), 889-893. https://doi.org/DOI: 10.1126/science.7754376

Siegel, J. S., Power, J. D., Dubis, J. W., Vogel, A. C., Church, J. A., Schlaggar, B. L., \& Petersen, S. E. (2013). Statistical improvements in functional magnetic resonance imaging analyses produced by censoring high-motion data points. Human Brain Mapping, 35(5), 
1981-1996. https://doi.org/10.1002/hbm.22307

Smith, S. M., Jenkinson, M., Woolrich, M. W., Beckmann, C. F., Behrens, T. E. J., JohansenBerg, H., ... Matthews, P. M. (2004). Advances in functional and structural MR image analysis and implementation as FSL. NeuroImage, 23, S208-S219.

https://doi.org/10.1016/j.neuroimage.2004.07.051

Thompson-Schill, S. L., D’ Esposito, M., Aguirre, G. K., \& Farah, M. J. (1997). Role of left inferior prefrontal cortex in retrieval of semantic knowledge : A reevaluation. Neurobiology, 94(December), 14792-14797.

Vazquez, A. L., \& Noll, D. C. (1998). Nonlinear aspects of the BOLD response in functional MRI. NeuroImage. https://doi.org/10.1006/nimg.1997.0316

Wagner, A. D., Paré-Blagoev, E. J., Clark, J., \& Poldrack, R. A. (2001). Recovering meaning: left prefrontal cortex guides controlled semantic retrieval. Neuron, 31(2), 329-338. Retrieved from http://www.ncbi.nlm.nih.gov/pubmed/11502262

Wheeler, M. E., \& Buckner, R. L. (2003). Functional Dissociation among Components of Remembering: Control, Perceived Oldness, and Content. Journal of Neuroscience, 23(9), 3869-3880. https://doi.org/10.1523/JNEUROSCI.23-09-03869.2003

Woodcock, R. W., \& Johnson, M. B. (2002). Woodcock-Johnson-Revised Tests of Achievement. Itasca, IL: Riverside Publishing.

Woolrich, M. W., Jbabdi, S., Patenaude, B., Chappell, M., Makni, S., Behrens, T., ... Smith, S. M. (2009). Bayesian analysis of neuroimaging data in FSL. NeuroImage, 45(1), S173S186. https://doi.org/10.1016/j.neuroimage.2008.10.055

Yonelinas, A. P., \& Levy, B. J. (2002). Dissociating familiarity from recollection in human recognition memory: different rates of forgetting over short retention intervals. Psychonomic Bulletin \& Review, 9(3), 575-582. Retrieved from http://www.ncbi.nlm.nih.gov/pubmed/12412899

Yonelinas, A. P., Otten, L. J., Shaw, K. N., \& Rugg, M. D. (2005). Separating the Brain Regions Involved in Recollection and Familiarity in Recognition Memory. The Journal of Neuroscience, 25(11), 3002-3008. https://doi.org/10.1523/JNEUROSCI.5295-04.2005 


\section{Supplementary Figures:}

Cingulo Parietal and Frontal-Parietal connectivity comparisons at other movement thresholds Pseudowords Words

FD threshold 0.25

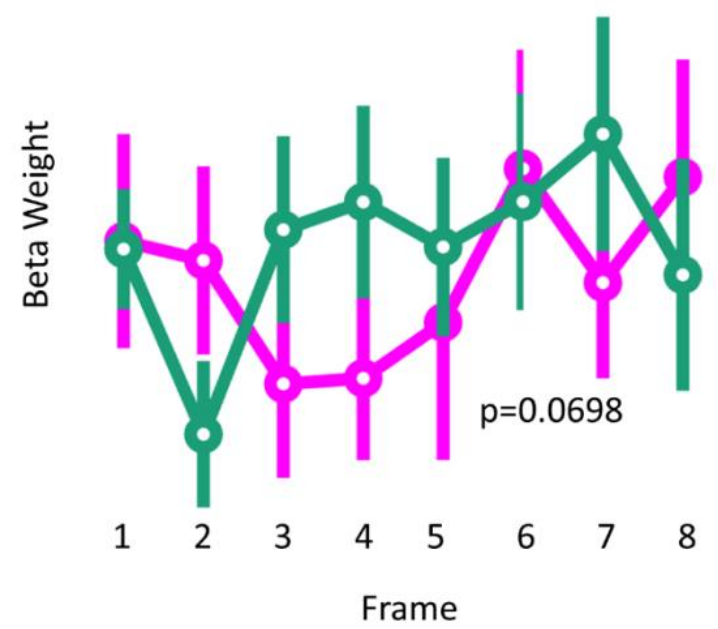

FD threshold 0.50

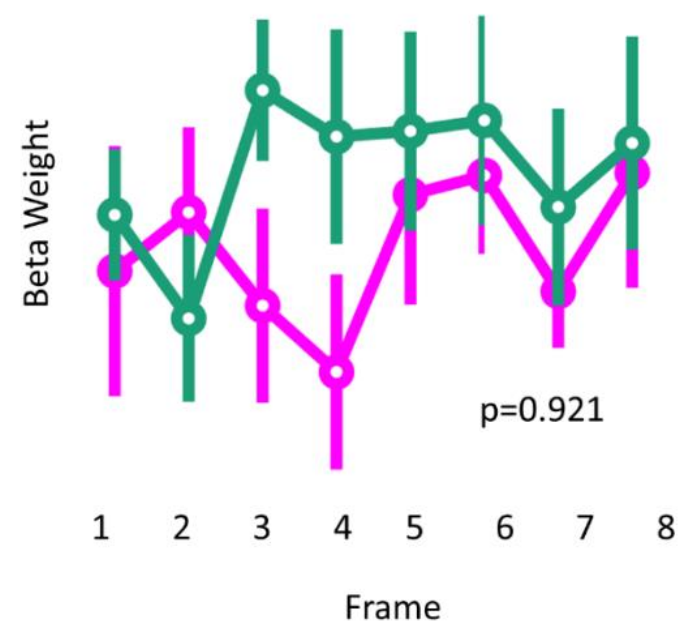

Figure 5 Supplement 1: Difference of how the Cingulo Parietal and Fronto Parietal networks interact over time at other thresholds of movement suggest robust initial finding. The left side shows the change in beta-weights between the Fronto-Parietal and Cingulo Parietal networks at a movement threshold of $0.25 \mathrm{~mm}$. After correcting for multiple comparisons, this functional system pair was not found to be $\operatorname{significant}(\mathrm{p}=0.0698)$. The right side shows the results from the same analysis as the left information when the movement threshold was set at a higher value of $0.5 \mathrm{~mm}$. When this data underwent corrections for multiple comparisons, this functional system pair was not found to be significant $(\mathrm{p}=0.921)$. 


\section{Network level connectivity differences across time and stimulus}

\section{using correlations}
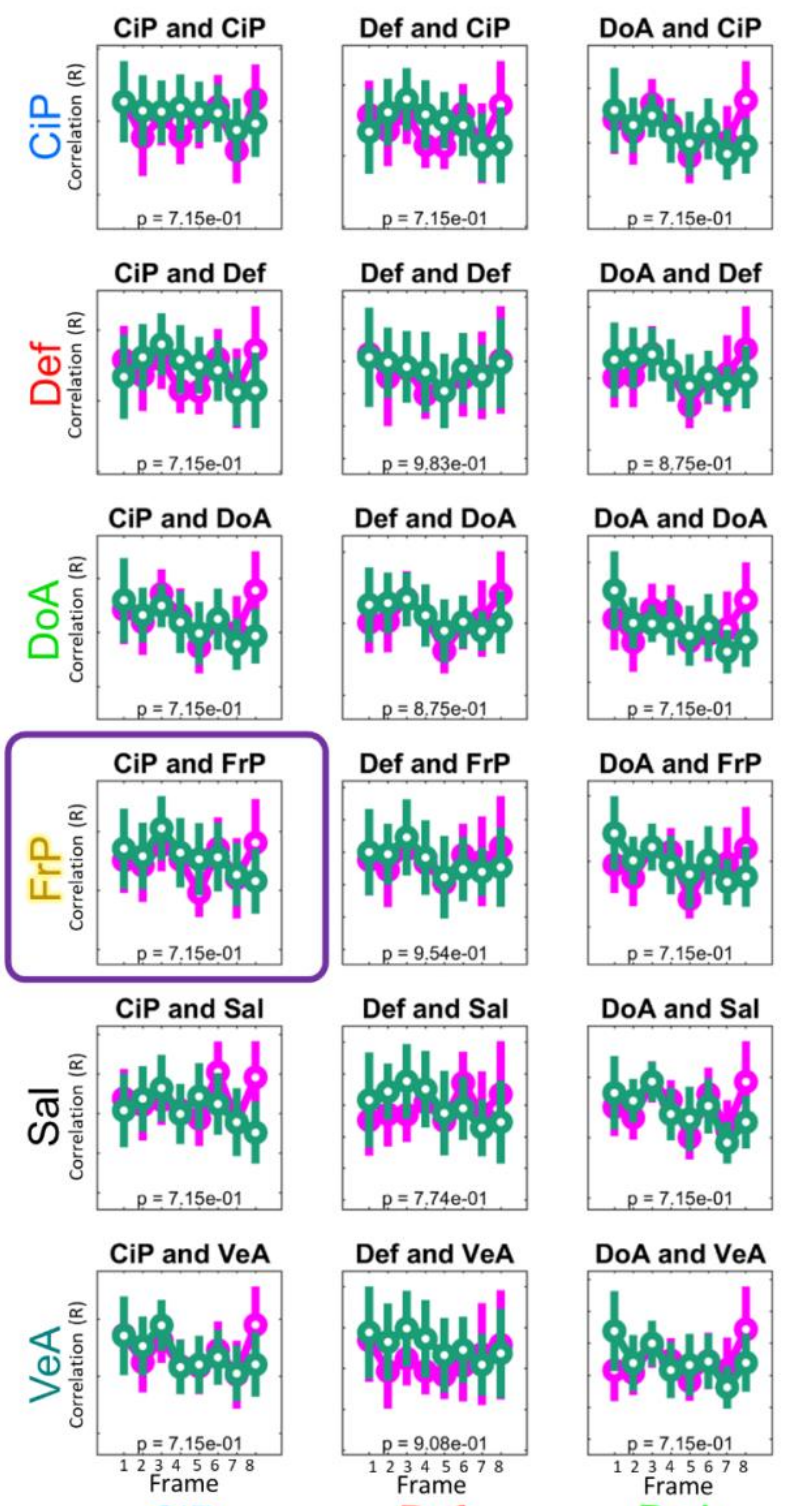

$\mathrm{CiP}$

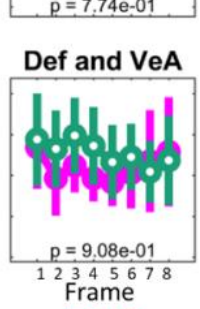

Def

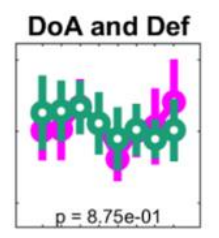

DoA and DoA
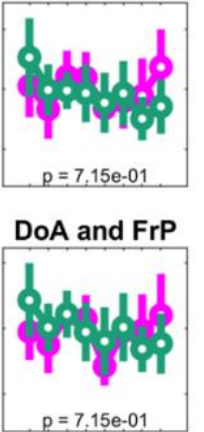

DoA and Sal

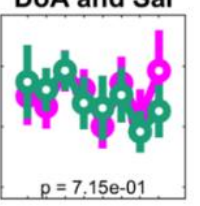

DoA and VeA

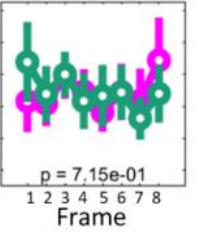

DoA

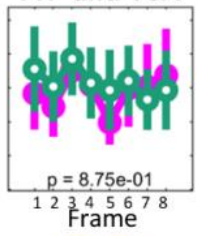

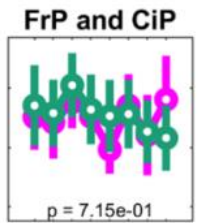
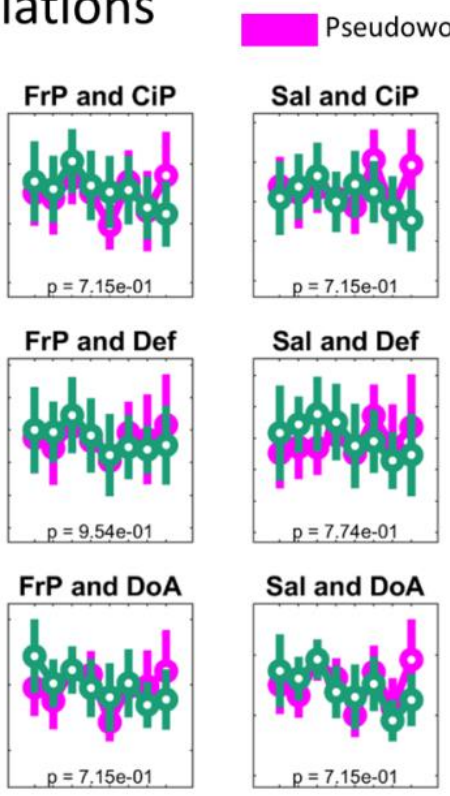

FrP and FrP
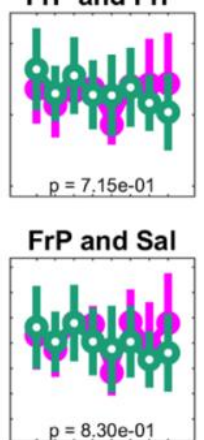

FrP and VeA

FrP

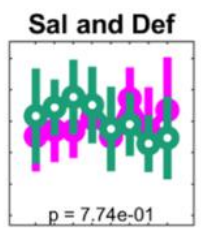

Sal and DoA

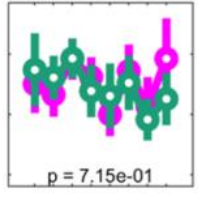

Sal and FrP
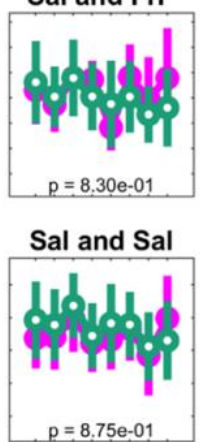

Sal and VeA

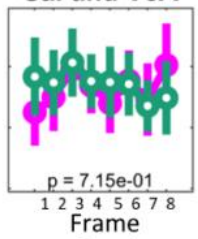

Sal
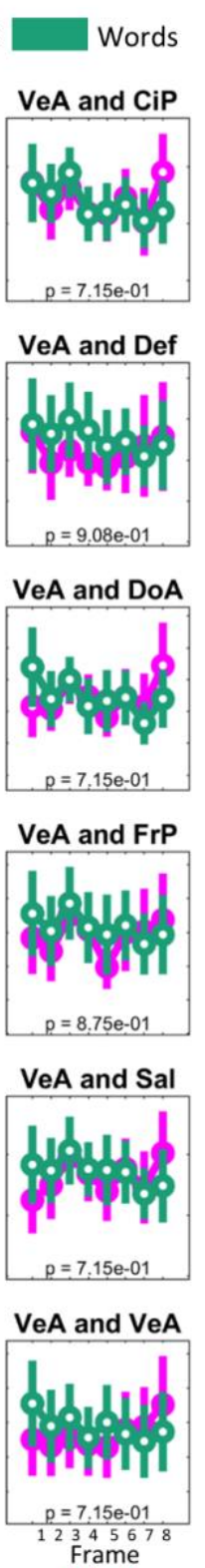

VeA

Figure 5 Supplement 2: Statistical analysis using traditional correlations did not reveal temporal differences.

Repeated measures ANOVA results on functional connectivity data characterized through the traditional correlation approach. 36 functional system pairs were tested within the following six networks: Auditory (Aud, n=24), Cingulo Opercular (CiO, n=40), Cingulo Parietal (CiP, n=5), Default (Def, $n=41$ ), Dorsal Attention (DoA, n=32), Fronto-Parietal (FrP, n=24), Retrosplenial Temporal (ReT, $\mathrm{n}=8)$, Somato-sensory hand $(\mathrm{Sml}, \mathrm{n}=38)$, Somato-sensory mouth $(\mathrm{SMm}, \mathrm{n}=8)$, Salience (Sal, n=4), Ventral Attention (VeA, n=23), and Visual (Vis, $n=39$ ) networks. The functional system pair found to be significant with connectotyping is outlined in purple but with this approach did not reveal similar findings. 